\title{
Análisis del uso y eficacia del globo para recuperar la red en función del contexto de juego en pádel Analysis of the use and effectiveness of lobs to recover the net in the context of padel *Diego Muñoz Marín, **Javier Courel Ibáñez, ***Bernardino J Sánchez-Alcaraz Martínez, *Jesús Díaz García, *Francisco Javier Grijota Pérez, *Jesús Muñoz Jiménez \\ *Universidad de Extremadura (España), **Universidad de Granada (España), ***Universidad de Murcia (España)
}

Resumen. El objetivo de este estudio fue analizar el tipo de acciones de subida a la red y su eficacia en función del nivel de competición y el contexto de juego en pádel. Se analizaron un total de 416 acciones de intercambio de posiciones (del fondo a la red) pertenecientes a 14 partidos de torneos masculinos: ocho finales del circuito profesional World Pádel Tour (WPT) y seis finales de un torneo de la Primera Categoría Federada Extremeña (CEP). De cada acción de intercambio, se analizó el tipo de golpeo previo a la subida de la red en zona defensiva distinguiendo entre el uso o no de globos. Se utilizaron regresiones logísticas binarias y el análisis de clasificación en árbol (CHAID) para determinar un modelo predictor del uso y la eficacia de las acciones para subir a la red. Los principales resultados muestran una prevalencia significativa en el uso del globo frente a otras acciones técnicas (60.6\% vs. 39.4\%), aumentando además la probabilidad de continuidad del punto (65.9\% vs. 47.0\%). No se encontraron influencias en las variables contextuales sobre el tipo de acción de subida a la red. Sin embargo, el análisis CHAID clasificó por orden de importancia las variables más influyentes en las acciones de subida a la red, sirviendo de guía para estudios futuros.

Palabras Clave: deportes de raqueta, táctica, toma de decisión, rendimiento, análisis del juego.

\begin{abstract}
The aim of this study was to analyse the use and effectiveness of actions to approach net in padel, based on competition level and game context. A total of 416 rallies (from baseline to net) were recorded from 14 male matches: eight finals of World Padel Tour (WTP) tournaments and six championship matches of the Extremadura Padel Federation first category (CEP) circuit. The analysis included baseline shots performed before approaching the net, categorizing them depending on the use/non-use of lobs. Binary logistic regression and tree classification analyses (CHAID) were used to predict the use and effectiveness of the actions carried out to approach the net. Main results revealed a significant prevalence of lobs compared to other types of shots (60.6 \% vs. 39.4\%); further, performing lobs increased the likelihood of continuity of rallies (65.9\% vs. $47.0 \%)$. No influences of game contextual variables were observed. CHAID analysis provided with a ranking of variables that influence the most the action of approaching the net, which could represent a useful guideline for future studies.
\end{abstract}

Key Words: racquet sport, tactic, decision-making, performance, match analysis.

\section{Introducción}

El pádel, pese a ser un deporte de reciente creación y con escaso desarrollo científico, se está convirtiendo cada vez más en objeto de estudio por parte de investigadores. En concreto, existe un especial interés en la descripción de la competición y el descubrimiento de indicadores de rendimiento a través del análisis del juego (Courel-Ibáñez, 2015; Sánchez-Alcaraz, Cañas \& Courel-Ibáñez, 2015; Sánchez-Alcaraz, Sánchez-Pay, Gómez-Mármol, Bazaco, \& Molina, En prensa). Este tipo de análisis permite la extracción de datos a partir de comportamientos espontáneos y en contextos reales de competición, proporcionando información objetiva de situaciones reales de juego que resulta de gran interés para el diseño y programación de entrenamientos (Anguera, 2003; McGarry, O’Donoghue \& Sampaio, 2013). El análisis del rendimiento ha sido ampliamente estudiados en otros deportes de raqueta como el tenis (Martínez-Gallego et al., 2013) o el squash (CatalánEslava \& González-Villora, 2015).

En pádel, uno de los aspectos más estudiados ha sido la detección de indicadores que aumenten la eficacia de ganar el punto. Desde un punto de vista especial, estudios previos resaltan la importancia de ocupar y mantener posiciones cercanas a la red para aumentar las probabilidades de éxito (Courel, Sánchez-Alcaraz \& Cañas, 2015; RamónLlin, Guzmán, Llana, Vuckovic \& James, 2013; Torres-Luque, Ramirez, Cabello-Manrique, Nikolaidis \& Alvero-Cruz, 2015). Estos trabajos muestran que más del $80 \%$ de los puntos directos se obtienen desde la red, siendo además la volea (por ejemplo, golpe directo desde la red) el golpe más común, representando el 30\% del total de golpeos (TorresLuque et al., 2015). Por tanto, durante el desarrollo del punto, los jugadores que se encuentran en posiciones defensivas, luchan por recuperar la red y enviar a sus rivales al fondo de la pista, mientras que los jugadores que se encuentran en la red tratan de mantener su posición ventajosa (Courel-Ibáñez, Sánchez-Alcaraz \& Cañas, En prensa; Lasaga, 2010). Sin embargo, existe una importante carencia de estudios que analicen las acciones que realizan los jugadores de pádel durante el

Fecha recepción: 04-03-16. Fecha de aceptación: 24-05-16 Diego Muñoz Marín

diegomun@unex.es punto, con el objetivo de detectar aquellas conductas técnicas y tácticas que resulten más eficaces tanto para mantener como para recuperar la red, como por ejemplo, posicionamiento en el campo desde la red, dirección del resto a la derecha o al revés, secuencia de patrones de golpeo o desplazamiento de los jugadores (Ramón-Llín \& Guzmán, 2014).

Con el objetivo de poder acceder a posiciones ofensivas desde el fondo de la pista, los jugadores pueden realizar diferentes tipos de golpeos, presentándose el globo como la acción técnica más realizada (alrededor del 15\% de los golpes en pádel son globos), aunque parece que estos datos podrían variar en función de diferentes variables como el género (Torres et al., 2015) o la edad de los jugadores (Carrasco, Romero, Sañudo \& De Hoyo, 2011; Sañudo, De Hoyo \& Carrasco, 2008). Sin embargo, aunque son varias las investigaciones que han analizado la frecuencia y el tipo de golpeos en pádel (Almonacid, 2011; Carrasco et al., 2011; Sañudo et al., 2008; Torres et al., 2015), no se han encontrado estudios que analicen las diferentes acciones que realizan los jugadores para subir a la red y sus diferencias entre otras variables como el nivel de juego o el resultado del partido.

A nivel contextual, las investigaciones en pádel que han estudiado las diferencias según el nivel de juego han mostrado que los jugadores de nivel alto realizan más puntos por partido, más golpeos por punto, y que la duración de sus puntos es mayor (Sánchez-Alcaraz, 2014a; 2014b), así como una mayor precisión en sus golpes (Sánchez-Alcaraz, Courel-Ibáñez \& Cañas, 2016). Del mismo modo, los jugadores expertos recorren más distancia por partido y su velocidad de desplazamiento es mayor en comparación con jugadores noveles (Ramón-Llín, 2013; Ramón-Llín, et al., 2013). Además, se ha demostrado la asociación entre el «momentum» del partido y la realización de determinadas acciones técnicas en otros deportes de raqueta (Knight \& O’Donoghue, 2011; Moss \& O’Donoghue, 2015), no encontrando referencias de estudios que exploren estas influencias en pádel.

Por lo tanto, dada la importancia de lograr posiciones ofensivas en la red en pádel, y debido a la falta de investigaciones sobre variables situacionales en este deporte, el objetivo de este estudio será analizar el tipo de acciones de subida a la red y su eficacia en función del nivel de competición y el contexto de juego en pádel. 


\section{Método}

\section{Muestra}

Se analizaron un total de 416 acciones de intercambio de posiciones (del fondo a la red) pertenecientes a 14 partidos masculinos de dos competiciones diferentes, jugados en el año 2014 en categoría masculina y disputados al mejor de tres sets: ocho finales de torneos del circuito profesional World Pádel Tour (WPT) y seis finales de torneos de la Primera Categoría Federada Extremeña (CEP). Se han seleccionado de forma aleatoria solamente acciones de las rondas finales de los torneos, asegurando la mayor igualdad entre las parejas y el máximo nivel competitivo hasta el final del partido (Mesagno, Geukes \& Larkin, 2015). Se consideraron como acciones de intercambio cuando la pareja que realiza la acción golpea inicialmente en el fondo de la pista (zona defensiva), y posteriormente en la red (zona ofensiva). La zona de red se delimitó situando la referencia visual de la reja horizontal situada a cuatro metros de la red(Figura 1, extraído de Courel et al., 2015; RamónLlin y Guzmán, 2014).

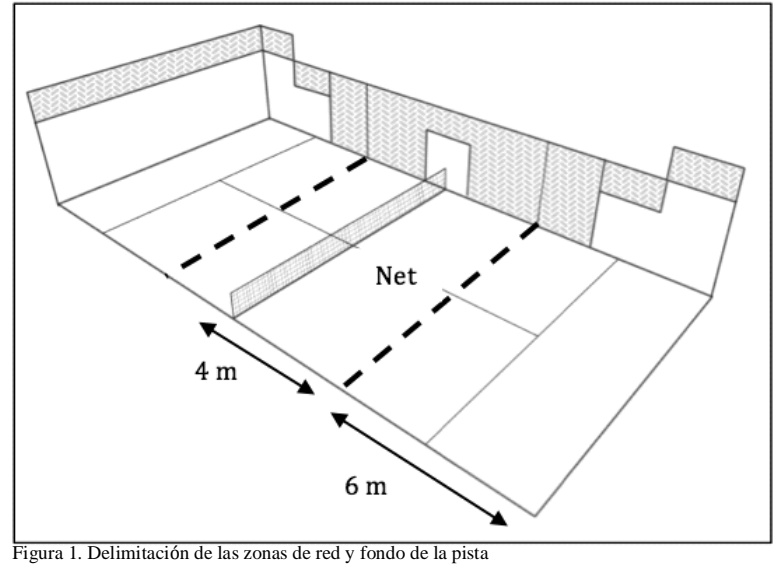

\section{Variables}

De cada acción de intercambio, se analizó el tipo de golpeo previo a la subida de la red en zona defensiva, distinguiendo entre globos (acción de enviar la pelota por encima de los jugadores rivales con el objetivo de sobrepasarles y obligarles a retroceder a posiciones defensivas de la pista) y no globos (acciones en donde la pelota no supera la altura de la cabeza de los rivales, como por ejemplo, bajadas de pared, voleas de contraataque o «chiquitas»). Además, se analizaron las siguientes variables relacionadas con el contexto de juego:

- Nivel de competición: distinguiendo entre CEP y WPT

- Set: distinguiendo entre primer, segundo y tercer set.

- Diferencia de juegos: se consideróla diferencia en el marcador en cuanto a juegos conseguidos por cada una de las parejas, estableciendo 3 categorías: iguales, diferencia $=$ un juego, diferencia $>$ un juego.

- Puntos en el marcador: determinado como diferencia de puntos en el marcador que se producen en un juego o tie- break. Por ejemplo, cuando el marcador se encuentra en 30-15, la diferencia en el marcador será uno; si es 40-15, será dos.

- Puntos clave: hace referencia a los puntos que pueden tener incidencia en el resultado del partido, es decir, son aquellos en los que cualquiera de las 2 parejas tienen opción de conseguir un juego, set 0 partido.

El rendimiento de cada acción de intercambio se midió a través del resultado de la acción consecutiva en la red, consideraron dos opciones: punto terminado (cuando el golpe fue punto ganador o error), y continuidad del punto (cuando el siguiente golpe no fue definitivo). Esta decisión fue tomada debido al insuficiente porcentaje de muestra con puntos ganadores ( $<10 \%$ del total), no siendo fiable la división de la variable en función del resultado del punto.

\section{Procedimiento}

Los datos fueron registrados mediante observación sistemática, a través del uso del software específico LINCE(Gabín, Camerino, Anguera \& Castañer, 2012). Dos observadores titulados y especializados en pádel fueron específicamente entrenados para esta tarea. Al término del proceso, cada observador analizó los mismos dos partidos ( $\mathrm{n}=72$ acciones de intercambio) con el objetivo de calcular la confiabilidad inter-observador a través del Multirater Kappa Free (Randolph, 2005), obteniendo valores por encima de .80. Para asegurar la consistencia de los datos, se evaluó la confiabilidad intra-observador al final del proceso de observación, obteniendo valores mínimos de.89. Siguiendo aAltman (1991, p.404), los valores de kappa obtenidos permitieron considerar el grado de acuerdo como muy alto (>.80). Todos los partidos de las finales analizadas de las acciones de CEP fueron grabados por técnicos de la Federación Extremeña de Pádel, con una cámara digital (SONY HDR-PJ620, Japón) situada a tres metros de altura y otros tres metros por detrás de uno de los cristales del fondo de pista. Dichos partidos son retransmitidos en streaming y posteriormente alojados en la página web de la Federación Extremeña de Pádel. Del mismo modo, las acciones correspondientes a la competición WPT fueron grabadas por la organización de WPT y alojadas también en la web correspondiente, donde han sido visualizadas para la observación, recogida y análisis de los datos.

\section{Análisis estadístico}

La descripción inicial de las variables incluyó el cálculo de distribución de frecuencias y porcentajes. En primer lugar, se utilizó un análisis de regresión logística binaria para predecir el efecto de las variables estudiadas sobre la acción de subida a la red, a través del cálculo de los Odds Ratio(OR) y el intervalo de confianza(IC) al 95\% de los predictores y estableciendo el nivel de significativos en $p<.05$ (Test de Wald). Posteriormente, se utilizó un análisis de clasificación en árbol para determinar un modelo predictor del uso de las acciones de subida hacia la red. Se recurrió al algoritmo CHAID (Chi Squared automatic interactions detection) exhaustivo para identificar las relaciones entre las variables independientes categóricas. El nivel de significación se estableció en $p<.05$, considerando un máximo de 100 interacciones y un cambio mínimo en las frecuencias esperadas de .001. Con el fin de obtener un modelo completo, se estableció un nivel de significación para la división de nodos en .100. La fuerza de asociación de las variables y el tamaño del efecto se estudiaron calculando el pseudo-R2 de Cox \& Snell y de Nagelkerke para los análisis de regresión, y recurriendo a los Residuos Tipificados Corregidos (RTCs) y al coeficiente Phi (ö) para los análisis de Chi Cuadrado (Fritz, Morris \& Richler, 2012). Dado el carácter impredecible de las acciones de juego, y a la influencia del ambiente de competición en las interacciones de los jugadores, las observaciones se consideraron como independientes (Duarte, Araujo, Correia \& Davids, 2012). Los análisis se realizaron con el programa IBM SPSS v. 20.0 para Macintosh (Armonk, NY: IBM Corp.).

\section{Resultados}

La tabla 1 muestra la distribución de frecuencias y porcentajes de las variables estudiadas. Se registraron prácticamente el mismo número

\begin{tabular}{lcc}
\hline Tabla 1. & & \\
Distribución de las frecuencias y porcentajes de las variables estudiadas & \\
\hline Variables & n & $\%$ \\
\hline Acción de subida & 252 & 60.6 \\
$\quad$ Globo & 164 & 39.4 \\
$\quad$ No globo & & \\
Resultado acción en la red & 117 & 28.2 \\
$\quad$ Termina el punto & 299 & 71.8 \\
$\quad$ Continúa el punto & & \\
$\quad$ Nivel de competición & & 50.5 \\
$\quad$ Extremeña (CEP) & 210 & 49.5 \\
$\quad$ Profesional (WPT) & 206 & \\
Set & & 31.0 \\
$\quad$ Primer set & 129 & 43.8 \\
$\quad$ Segundo set & 182 & 25.2 \\
$\quad$ Tercer set & 105 & 36.5 \\
Diferencia de puntos & & 42.3 \\
Iguales & 152 & 21.2 \\
$\quad$ Diferencia 1 punto & 176 & 72.4 \\
$\quad$ Diferencia 2 o más puntos & 88 & 27.6 \\
$\quad$ Punto clave & & \\
$\quad$ Punto no decisivo & & \\
Punto decisivo & 301 & \\
\hline
\end{tabular}


de acciones en los partidos analizados. Sin embargo, observamos un predominio del uso del globo como acción de subida a la red y una tasa de continuidad del punto de cerca del 70\%.

La tabla 2 refleja los resultados del análisis de regresión logística binaria, considerando como variable dependiente la acción de subida a la red. Se encontró que la probabilidad de continuar el punto después de usar el globo fue entre 1.6 y 4.8 veces mayor en comparación con el uso de acciones a media o baja altura $(\mathrm{X} 2(11)=25.810 ; p=.007 ; \mathrm{R} 2: .07-.10)$. No se observaron efectos de ninguna variable contextual.

La figura 2 muestra el modelo de análisis CHAID de clasificación de

Tabla 2.

\begin{tabular}{|c|c|c|c|c|c|}
\hline \multicolumn{6}{|c|}{ Acción de subida a la red (Ref: Globo) } \\
\hline \multirow{4}{*}{\begin{tabular}{|l|}
\multicolumn{1}{c|}{ Predictores } \\
Resultado acción en la red (a) \\
Nivel de competición (b) \\
Set (c)
\end{tabular}} & \multirow{2}{*}{\multicolumn{2}{|c|}{\begin{tabular}{c||c}
\multicolumn{2}{c||}{ Accon de subi } \\
$\boldsymbol{B}$ & $\boldsymbol{p}$ \\
1.05 & $<. \mathbf{0 0 1}^{*}$
\end{tabular}}} & \multirow{2}{*}{\begin{tabular}{|c|} 
OR \\
2.771
\end{tabular}} & \multicolumn{2}{|c|}{ IC $95 \%$} \\
\hline & & & & $1.601-$ & 4.797 \\
\hline & -.37 & .137 & .687 & $.419-$ & 1.126 \\
\hline & & & & & \\
\hline Set 1 & -.44 & .169 & .639 & $.337-$ & 1.210 \\
\hline Set 2 & -.08 & .774 & .917 & $.510-$ & 1.651 \\
\hline Diferencia de puntos (d) & & & & & \\
\hline Iguales & -.93 & 132 & .394 & $.117-$ & 1.322 \\
\hline Diferencia de un punto & -.67 & .258 & .332 & $.106-$ & 1.044 \\
\hline Puntos clave (e) & .29 & .392 & .509 & $.158-$ & 1.639 \\
\hline Diferencia de juegos & .17 & .114 & 1.192 & $.959-$ & 1.482 \\
\hline
\end{tabular}

árbol sobre las acciones de subida a la red. Únicamente encontramos una asociación significativa con el resultado de la acción en la red (X2(1)=12,549; $p=.004$; RTCs= 3.4; $\ddot{o}=.20$ ), obteniendo un $53.0 \%$ de casos en los que punto termina cuando no se usa globo (Nodo 1 ) frente a un $65.9 \%$ de casos en los que el punto continúa tras globo (Nodo 2). Aunque el resto de asociaciones no fueron significativas, encontramos un leve aumento de los puntos terminados tras no globo en la competición CEP frente a WPT (Nodo 3). Igualmente, se observa un ligero aumento en el uso del globo cuando el punto continuó (Nodo 2), en puntos no decisivos (Nodo 5) con un marcador igualado o con diferencia de un juego en el marcador (Nodo 7).

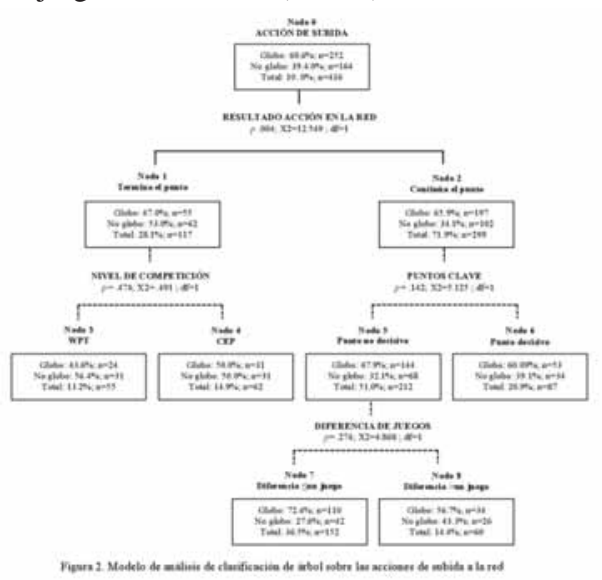

\section{Discusión}

El objetivo de este estudio fue analizar el tipo de acciones de subida a la red y su eficacia en función del nivel de juego y de las variables contextuales de los jugadores de pádel. Los principales resultados muestran una prevalencia significativa en el uso del globo frente a otras acciones técnicas, aumentando además la probabilidad de continuidad del punto. Aunque no se encontraron influencias en las variables contextuales sobre el tipo de acción de subida a la red, el análisis CHAID de clasificación de árbol permite el desglose de posibles relaciones potenciales de estudio en el futuro. Este método ofrece una visión novedosa hasta el momento en el estudio del análisis de la competición en pádel, siendo de gran interés para la extracción de conclusiones en este tópico de investigación.

En función del rendimiento de la acción de intercambio posterior a la acción de subida a la red, se encontró que la probabilidad de continuar el punto después de usar el globo fue significativamente más elevada en comparación con el uso de acciones a media o baja altura. De acuerdo con Almonacid (2011) y Priego et al., (2013), en pádel profesional el 25$30 \%$ de los golpeos corresponden a globos, realizados en su mayoría desde el fondo de la pista. Sin embargo, nuestros resultados muestran un aumento considerable en éstos porcentajes cuando el objetivo es alcanzar la red, incluyéndose en seis de cada diez acciones de subida a la red. Por otro lado, estudios previos han encontrado diferencias en el número de acciones en función del nivel y categoría de juego, registrando un mayor número de golpes por punto en jugadores profesionales en comparación a jóvenes jugadores de pádel (Sánchez-Alcaraz, 2014a; 2014b). En el presente estudio se ha observado un número de similar de progresiones hacia la red en jugadores senior profesionales y semiprofesionales. Estos resultados no obstante podrían sugerir que las diferencias en el rendimiento del juego en la red, como por ejemplo la situación de los jugadores en la red, tiempo mantenido en la zona ofensiva, o las trayectorias y velocidades de los golpeos en la red (Espino, Skiadopoulos, Gianikellis, \& Luís del Campo, 2016; Ramón-Llín, Guzmán, Llana, \& Vuckovic, 2016), podrían ser las determinantes del nivel de los jugadores.

El uso del globo parece propiciar mejores condiciones para subir a la red en jugadores expertos, ya que la correcta ejecución de este tipo de golpe provoca que los rivales golpeen en situaciones lejanas a la red (Sánchez-Alcaraz, 2013). Por otro lado, utilizar golpeos planos o cercanos a la cinta de la red para propiciar una acción de intercambio de los cuatro jugadores en posiciones de ataque que supondría una situación más definitiva para el desenlace del punto. Estos resultados ponen de manifiesto la importancia de uno de los principales objetivos tácticos del pádel, en el que se destaca la toma de decisión en la elección del golpe en función de la zona de la pista, con el objetivo de reducir al máximo el número de errores (Barberó, 2007; Sánchez, 2009; Sánchez-Alcaraz, 2013).

Por otro lado, pese a que no se han observado influencias significativas de las variables contextuales en el tipo de acciones de subida a la red, los resultados parecen sugerir que los jugadores de menor nivel utilizarían un mayor número de globos, mientras que los jugadores profesionales variarían más entre las acciones de subida a la red. Estos datos están en línea con otras investigaciones en tenis que han mostrado que deportistas más avanzados presentan un mayor nivel de recursos tácticos (Del Villar, García, Iglesias, Perla \& Cervelló, 2007; García, 2006; García, Moreno, Moreno, Iglesias, \& Del Villar, 2008).

Un resultado interesante ha sido el ligero aumento en el uso del globo cuando el punto continuó, en puntos no decisivos, con un marcador igualado o con diferencia de un punto en el marcador, indicando que los jugadores de pádel adoptan un estilo de juego más defensivo cuando el marcador es más igualado. Estos datos parecen estar en conexión con el estudio de Courel-Ibáñez \& Sánchez-Alcaraz (En prensa) que mostraron una gran prevalencia de errores en los puntos de corta duración (menos de cuatro segundos), y a medida que el punto avanza, los jugadores cometen menos errores. Además, estudios pilotos han mostrado efectos del tiempo de descanso (por ejemplo, intervalo entre puntos) y la eficacia del punto en momentos clave del partido (Díaz, García, Grijota, Muñoz, \& Muñoz, 2015). Estos resultados sugieren cambios fisiológicos favoreciendo la recuperación del jugador, así como un medio táctico y psicológico para afrontar mejor el punto en momentos decisivos. Por lo tanto, aunque estas asociaciones en el modelo no fueron significativas, conviene señalarlas como potenciales para su exploración en análisis futuros.

Este estudio presenta ciertas limitaciones que requieren ser tenidas en cuenta a la hora de interpretar los resultados. En primer lugar, no se han estudiado de manera pormenorizada el tipo de golpeos utilizados para recuperar la red debido a la falta de referencias que nos den con precisión una definición del tipo de golpeos específicos en pádel. Igualmente, dado el reducido tamaño muestral, no ha sido posible determinar relaciones significativas en el modelo de árbol más allá del primer nodo. Del mismo modo, no ha sido posible analizar en profundidad variables de rendimiento del juego (por ejemplo, ganador o perdedor del punto) o específicas del golpeo (por ejemplo, golpe ganador, error forzado o error 
no forzado). En un futuro, como continuidad a este trabajo, sería interesante ampliar el análisis del comportamiento de los jugadores tanto para recuperar la red como una vez dispuestos en zona ofensiva (por ejemplo, cuando suba a la red un miembro o los dos miembros de la pareja) explorando además variables temporales, espaciales, situacionales y de rendimiento.

En conclusión, el uso del globo se presenta como la acción técnica más utilizada por los jugadores para alcanzar posiciones ofensivas en pádel, aunque los jugadores profesionales varían más las acciones de subida a la red que los jugadores de menos nivel. En función de las variables contextuales, se observa un ligero aumento en el uso del globo cuando el punto continuó, en puntos no decisivos, con un marcador igualado o con diferencia de un punto en el marcador.

\section{Referencias}

Altman, D. G (1991). Practical Statistics for Medical Research. London: Chapman \& Hall.

Anguera, M.T. (2003). Observational Methods (General). En R. Fernaìndez-Ballesteros (Ed.). Encyclopedia of Psychological Assessment. London: Sage.

Almonacid, B. (2011). Perfil de juego en pádel de alto nivel. Tesis Doctoral. Universidad de Jaén.

Barberó, G (2007). Didáctica de una clase de pádel. Retos, Nuevas Tendencias en Deporte, Educación Física y Recreación, 12, 54-57.

Carrasco, L., Romero, S., Sañudo, B., \& De Hoyo, M. (2011). Game analysis and energy requirements of paddle tennis competition. Science \& Sport, 26(6), 338-344.

Catalán-Eslava, M., \& González-Villora, S. (2015). Validación de un instrumento de evaluación en deportes de red-muro: squash (HERS). Retos, 27, 63-80.

Courel-Ibáñez, J. (2015). Análisis de la competición en pádel. En J. Courel, B. Sánchez-Alcaraz, J. Cañas \& R. Guerrero (Ed.), Investigación en Pádel, Volumen I (pp. 7-23). Murcia: Editum.

Courel-Ibáñez, J., Sánchez-Alcaraz, B. J., \& Cañas, J. (2015). Effectiveness at the net as a predictor of final match outcome in professional padel players. International Journal of Performance Analysis in Sport, 15(2), 632-640.

Courel-Ibáñez, J. \& Sánchez-Alcaraz, B.J. (En prensa). Efectos de las variables situacionales sobre la duración y eficacia de los puntos en jugadores de pádel de élite. Apunts, Educación Física y Deportes.

Courel-Ibáñez, J., Sánchez-Alcaraz, B.J., \& Cañas, J. (En prensa). Game performance and length of rally in profesional padel players. Journal of Human Kinetics.

Del Villar, F., García, L., Iglesias, D., Moreno, M., \& Cervelló, E. (2007). Expert-novice differences in cognitive and execution skills during tennis competition. Perceptual and Motor Skills, 104(2), 355-365.

Díaz, J., García, A., Grijota, F., Muñoz, J., \& Muñoz, D. (2015). Incidencia de la importancia del punto sobre variables temporales en pádel de primera categoría regional. Revista Andaluza de Medicina del Deporte, 8(4), 187.

Duarte, R., Araújo, D., Correia, V., \& Davids, K. (2012). Sports Teams as Superorganisms. Sports Medicine, 42(8), 633-642.

Espino, C., Skiadopoulos, Gianikellis, K., \& Luís del Campo, V. (2016). Análisis espacio-temporal de los golpes de pádel: salida de pared, bandeja y remate por tres. En J. Courel-Ibáñez, B.J. Sánchez Alcaraz \& J. Cañas (Ed.). Innovación e investigación en pádel (pp. 213219). Sevilla: Wanceulen.

Fritz, C. O., Morris, P. E. and Richler, J. J. (2012) Effect size estimates: Current use, calculations, and interpretation. Journal of Experimental Psychology: General, 141(1), 2-18.

Gabín, B., Camerino, O., Anguera, M.T., \& Castañer, M. (2012), Lince: multiplatform sport analysis software. Procedia Computer Science Technology, 46, 4692-4694.

García, L. (2006). El conocimiento táctico en tenis. Un estudio con jugadores expertos y noveles. Cuadernos de Psicología del Deporte, 6(2), 11-20.

García, L.; Moreno, M.P.; Moreno, A.; Iglesias, D., \& Del Villar, F. (2008). Análisis de las diferencias en el conocimiento de los jugadores de tenis en función del nivel de pericia deportiva. Motricidad, European Journal of Human Movement, 21, 31-53.
Knight, G, \& O’Donoghue, P. (2011). The probability of winning break points in Grand Slam men's singles tennis. European Journal of Sports Science, 12(6), 462-468.

Lasaga, M.J (2010). Estudio social y metodológico del pádel desde la percepción de técnicos y jugadores: una apuesta educativa. Tesis doctoral, Universidad de Sevilla.

Martínez-Gallego, R., Guzmán, J.F., James, N., Ramón-Llín, J., Crespo, M., \& Vuckovic, G. (2013). The relationship between the incidence of winners/errors and the time spent in different areas of the court in elite tennis. Journal of Human Sport \& Exercise, 8(3), 601-607.

McGarry, T. (2009). Applied and theoretical perspectives of performance analysis in sport: Scientific issues and challenges. International Journal of Performance Analysis in Sport, 9(1), 128-140.

McGarry, T., O’Donoghue, P., \& Sampaio, J. (2013). The Routledge Handbook of Sports Performance Analysis. Londres: Routledge.

Mesagno, C., Geukes, K., \& Larkin, P. (2015). Choking under pressure: A review of current debates, literature, and interventions. In S. D. Mellalieu \& S. Hanton (Eds.), Contemporary advances in sport psychology: A review. New York: Routledge.

Moss, B., \& O’Donoghue, P. (2015). Momentum in US Open men’s singles tennis. International journal of Performance Analysis in Sport, 15, 884-896.

Priego, J.I., Olaso, J., Llana-Belloch, S., Pérez-Soriano, P., González García, J.C. \& Sanchís, M. (2013). Padel: A Quantitative study of the shots and movements in the high performance. Journal of Human Sport \& Exercise, 8(4), 925-931.

Ramón-Llín, J. (2013). Análisis de la distancia recorrida y velocidad de desplazamiento en pádel. Tesis Doctoral: Universidad de Valencia.

Ramón-Llín, J., \& Guzmán, F. (2014). Distancia a la red de los jugadores de pádel en función del lado de juego. Revista Internacional de Deportes Colectivos, 18, 105-113.

Ramón-Llín, J. Guzmán, J.F., Llana, S., \& Vuckovic, G. (2016). Comparación del tiempo de permanencia en zona ofensiva entre jugadores ganadores y perdedores de pádel. En: J. Courel-Ibáñez, B.J. Sánchez Alcaraz \& J. Cañas (Ed.). Innovación e investigación en pádel (pp. 155-163). Sevilla: Wanceulen.

Ramón-Llín, J., Guzmán, J.F., Llana, S., Vuckovic, G., \& James, N. (2013). Comparison of distance covered in paddle in the serve team according to performance level. Journal of Human Sport \& Exercise, 8(3), 738-742.

Randolph, J. J. (2005). Free-marginal multirater kappa: An alternative to Fleiss' fixed-marginal multirater kappa. Comunicación presentada en el Joensuu University Learning and Instruction Symposium 2005, Joensuu, Finlandia.

Sánchez, M. J. (2009). Metodología del Pádel en la Educación Física Escolar. Revista Digital Innovación y Experiencias Educativas, 23, 1-9.

Sánchez-Alcaraz, B. J. (2013). Táctica del pádel en la etapa de iniciación. Trances, Revista de Transmisión del Conocimiento Educativo y de la Salud, 5(1), 109-116.

Sánchez-Alcaraz, B. J. (2014a). Análisis de la exigencia competitiva del pádel en jóvenes jugadores. Kronos, 13(1), 1-7.

Sánchez-Alcaraz, B. J. (2014b). Diferencias en las acciones de juego y la estructura temporal entre el pádel masculino y femenino profesional. Acción Motriz, 12, 17-35.

Sánchez-Alcaraz, B. J., Cañas, J., \& Courel-lbáñez, J. (2015). Análisis de la investigación científica en pádel. AGON Revista International Journal of Sport Sciences, 5(1). 44-54.

Sánchez-Alcaraz, B.J., Courel-Ibáñez, J. \& Cañas, J. (2016). Valoración de la precisión del golpeo en jugadores de pádel en función de su nivel de juego. RICYDE, Revista Internacional de Ciencias del Deporte, 45(12), 324-333.

Sánchez-Alcaraz, B.J., Sánchez-Pay, A., Gómez-Mármol, A., Bazaco, M.J., \& Molina, J. (En prensa). Diferencias en la forma de organización de las sesiones de pádel en estudiantes. Revista Internacional de Medicina y Ciencias del Deporte.

Sañudo, B., De Hoyo, M., \& Carrasco, L. (2008). Demandas fisiológicas y características estructurales de la competición en el pádel masculino. Apunts. Educación Física y Deportes, 4, 23-28.

Torres-Luque, G., Ramirez, A., Cabello-Manrique, D., Nikolaidis, P. T., \& Alvero-Cruz, J. R. (2015). Match analysis of elite players during paddle tennis competition. International Journal of Performance Analysis in Sport, 15(3), 1135-1144. 